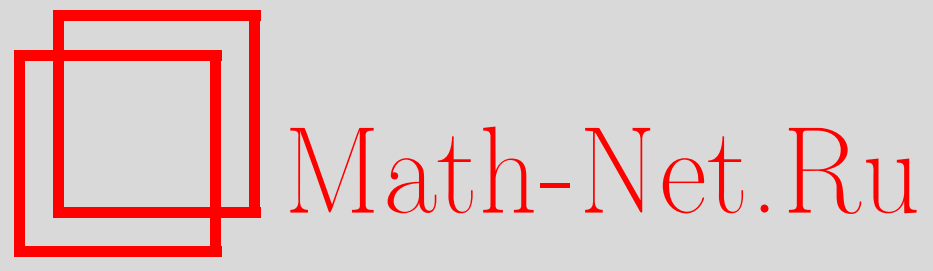

А. Ю. Кораблев, Е. И. Михайловский, Е. В. Тулубенская, Н. А. Беляева, Продольная устойчивость ребристой оболочки в разномодульной упругой среде, Вестн. Сам. гос. техн. ун-та. Сер. Физ.-мат. науки, 2014, выпуск 2(), 89-95

DOI: https://doi.org/10.14498/vsgtu1278

Использование Общероссийского математического портала MathNet.Ru подразумевает, что вы прочитали и согласны с пользовательским соглашением

http://www.mathnet.ru/rus/agreement

Параметры загрузки:

IP : 34.239 .49 .27

26 апреля 2023 г., 18:15:30

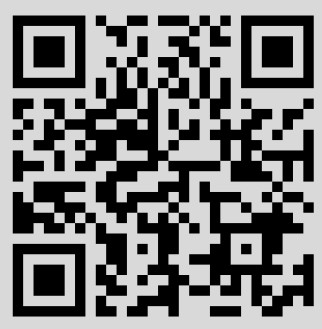


Вестн. Сам. гос. техн. ун-та. Сер. Физ.-мат. науки. 2014. № 2 (35). С. $89-95$

УДК 539.311

\title{
ПРОДОЛЬНАЯ УСТОЙЧИВОСТЬ РЕБРИСТОЙ ОБОЛОЧКИ В РАЗНОМОДУЛЬНОЙ УПРУГОЙ СРЕДЕ
}

\author{
А. Ю. Кораблев, Е. И. Михайловский, \\ Е. В. Тулубенская, Н. А. Беляева
}

Сыктывкарский государственный университет,

Россия, 167000, Сыктывкар, Октябрьский проспект, 55.

\begin{abstract}
Рассматривается задача об устойчивости продольно сжсимаемой шарнирно опёртой цилиндрической оболочки, подкреплённой стрингерами и расположенной на гранище двух упругих сред различной жёсткости. Вывод уравнений производился при следующих допущениях: применяется упрощённая теория Доннела-Власова, дебормация осесимметрична, стрингеры изгибаются в нормальной плоскости как гибкие стержни, на оболочку действует лишь нормальная нагрузка. Решение задачи ищется с помощъю комбинированного алгоритма перебора вариантов. Суть метода заключается в последовательном применении алгоритмов полного и локального перебора вариантов. Алгоритм полного перебора вариантов предназначен для построения качественно адекватной формы, после чего для уточнения значения критической силь применяется локальный метод перебора вблизи корней приближения $\kappa$ искомой собственной форме. $B$ результате проведенного численного эксперимента выяснено, что при увеличении количества стрингеров прочность оболочки повышается. Полученные результаты согласуются с результатами, полученными к настоящему времени в других paботах.
\end{abstract}

Ключевые слова: устойчивость, шарнирно-опёртая иилиндрическая оболочка, комбинированный алгоритм перебора вариантов.

Введение. В работе [1] описана так называемая деформационная теория ребристых оболочек, главная особенность которой заключается в том, что в ней впервые наряду с реактивной силой учтён реактивный момент от ребра жёсткости. Названная теория подробно изложена в монографии [2]. В частности, уравнения статики конструктивно ортотропной цилиндрической оболочки, получаемые путём «размазывания» регулярной системы стрингеров,

ISSN: 2310-7081 (online), 1991-8615 (print); doi: http://dx.doi.org/10.14498/vsgtu1278 (C) 2014 Самарский государственный технический университет.

Образец цитирования: А. Ю. К ор абл ев, Е. И. М и х ай л о в с к и й, Е. В. Т ул убен с к а я, Н. А. Б ел я е в а, "Продольная устойчивость ребристой оболочки в разномодульной упругой среде" // Вестн. Сам. гос. техн. ун-та. Сер. Физ.-мат. науки, 2014. № 2 (35). С. 89-95. doi: $10.14498 /$ vsgtu1278.

Сведения об авторах: Кораблев Анатолий Юръевич, аспирант, каф. математического моделирования и кибернетики. Евгений Ильич Михайловский (12.07.1937-11.07.2013) (д.ф.м.н., проф.). Елена Владимировна Тулубенская (к.ф.-м.н., доц.), доцент, каф. математического моделирования и кибернетики. Надежда Александровна Беляева (д.ф.-м.н., проф.), профессор, каф. математического моделирования и кибернетики.

E-mail addresses: astroori@mail.ru (A.Yu. Korablev, Corresponding author), vetamile@rambler.ru (E.V. Tulubenskaya), belyayevana@mail.ru (N.A. Belyaeva) 
имеют вид

$$
c_{0} \mathbf{L} u=-R^{2} q+\frac{1}{l}\left[\frac{K_{\nu}}{R^{2} \frac{\partial^{4} w}{\partial \xi^{4}}+\frac{K_{t}}{R^{2}} \frac{\partial^{4} w}{\partial \xi^{2} \partial \varphi^{2}}-\frac{\partial^{2} u_{1}}{R^{2}} \frac{\partial^{3} u_{2}}{\partial \xi^{2} \partial \varphi}}\right]
$$

Здесь первая строка уравнения представляет собой матричную запись уравнений статики цилиндрической оболочки в смещениях; $K_{\nu}, K_{n}$-жёсткости стрингера при изгибе соответственно в нормальной плоскости и из этой плоскости; $K_{t}$-жёсткость при кручении; $C_{t}$-жёсткость стрингера при растяжении (сжатии); $q_{1}, q_{2}, q_{n}$ - нагрузка; $l$ - расстояние между соседними стрингерами по дуге поперечного сечения; $\xi=x / R ; \varphi=y / R ; R$ - радиус оболочки.

Будем рассматривать оболочку, расположенную на границе двух винклеровских сред различной жёсткости. Примем следующие допущения:

i) для расчёта оболочек без рёбер допустимо использовать упрощённую теорию Доннела-Власова [3, табл. 13.2, форм. (13.18c)];

ii) конструктивно ортотропная оболочка испытывает осесимметричную деформацию, т. е. $\partial f / \partial \varphi=0$ для любой функции $f$;

iii) стрингеры изгибаются в нормальной плоскости как гибкие стержни, T. e.

$$
C_{t} \frac{\partial^{2} u_{1}}{\partial \xi^{2}}=\frac{K_{n}}{R^{2}} \frac{\partial^{4} u_{2}}{\partial \xi^{4}}=0
$$

iv) на оболочку действует лишь нормальная нагрузка, т. е. $q_{1}=q_{2}=0$.

Тогда уравнению (1) можно придать вид

$$
\mathbf{A}\left[\begin{array}{l}
u_{1} \\
u_{2} \\
w
\end{array}\right]=\frac{R^{2}}{c_{0}}\left[\begin{array}{c}
0 \\
0 \\
q_{n}
\end{array}\right], \quad c_{0}=\frac{E h}{1-\nu^{2}},
$$

где

$$
\mathbf{A}=\left[\begin{array}{ccc}
\frac{d^{2}()}{d \xi^{2}} & 0 & -\nu \frac{d()}{d \xi} \\
0 & \frac{1-\nu}{2} \frac{d^{2}()}{d \xi^{2}} & 0 \\
-\nu \frac{d()}{d \xi} & 0 & a_{33}
\end{array}\right], \quad a_{33}=\left(\frac{1}{12} \frac{h^{2}}{R^{2}}+\frac{\left(1-\nu^{2}\right) J}{l h R^{2}}\right) \frac{d^{4}()}{d \xi^{4}}+I
$$

$I$ - тождественный оператор, $J$ - момент инерции поперечного сечения стержня, $h$ - толщина неподкреплённой оболочки, $E$ - модуль Юнга, $\nu$ - коэффициент Пуассона материала ребристой оболочки.

Для получения из системы (2) соответствующего уравнения относительно функции прогиба воспользуемся операторным методом [4].

«Детерминант» матрицы А имеет вид

$$
\operatorname{det} \mathbf{A}=\frac{1-\nu}{2}\left(\frac{h^{2}}{12 R^{2}}+\frac{\left(1-\nu^{2}\right) J}{\operatorname{lh} R^{2}}\right) \frac{d^{8}()}{d \xi^{8}}+\frac{1-\nu}{2}\left(1-\nu^{2}\right) \frac{d^{4}()}{d \xi^{4}} .
$$


Заменяя в матрице А последний столбец правой частью уравнения (2) и вычисляя «детерминант» так составленной матрицы, получим

$$
\operatorname{det} \mathbf{A}_{w}=\frac{1-\nu}{2} \frac{R^{2}}{c_{0}} \frac{d^{4} q_{n}}{d \xi^{4}}
$$

Применяя формально правило Крамера, можно записать $w=\operatorname{det} \mathbf{A}_{w} / \operatorname{det} \mathbf{A}$ или $(\operatorname{det} \mathbf{A}) w=\operatorname{det} \mathbf{A}_{w}$. Последнее равенство после элементарных преобразований представляет искомое уравнение в виде

$$
\left(1+\frac{E J}{l d_{0}}\right) \frac{d^{4} w}{d \xi^{4}}+4 b^{4} w=\frac{R^{4}}{d_{0}} q_{n}
$$

где

$$
4 b^{4}=12\left(1-\nu^{2}\right) \frac{R^{2}}{h^{2}}, \quad d_{0}=\frac{E h^{3}}{12\left(1-\nu^{2}\right)} .
$$

Представим нормальную нагрузку в виде суммы

$$
q_{n}=q_{n}^{\prime}+q_{n}^{\prime \prime}
$$

В условиях наличия внутри и вне оболочки винклеровых сред различной жёсткости $c_{1}, c_{2}$ соответственно, следуя [5], можно записать $q_{n}^{\prime}=-c_{1} w_{+}-$ $-c_{2} w_{-}$. Кроме этого, при рассмотрении продольной устойчивости цилиндрической оболочки от действия сжимающих усилий $T_{0}$ следует положить [3]

$$
q_{n}^{\prime \prime}=-\frac{T_{0}}{R^{2}} \frac{d^{2} w}{d \xi^{2}}
$$

Выполним замену

$$
\tilde{\xi}=\frac{\pi R}{L} \xi=\frac{\pi R}{L} \frac{x}{R}=\frac{\pi x}{L} \in[0, \pi],
$$

где $L$ - длина оболочки, и подставим соотношения (4) в уравнение (3), сохранив за новой переменной $\tilde{\xi}$ прежнее обозначение $\xi$, окончательно получим

$$
(1+\alpha) \frac{d^{4} w}{d \xi^{4}}+4 \beta^{4} w+\lambda \frac{d^{2} w}{d \xi^{2}}+k_{1} w_{+}+k_{2} w_{-}=0 .
$$

Здесь

$$
\alpha=\frac{E J}{l d_{0}}, \lambda=\frac{T_{0} L^{2}}{\pi^{2} d_{0}}, 4 \beta^{4}=\frac{12\left(1-\nu^{2}\right) L^{4}}{\pi^{4} R^{2} h^{2}}, d_{0}=\frac{E h^{3}}{12\left(1-\nu^{2}\right)}, k_{i}=\frac{c_{i} L^{4}}{\pi^{4} d_{0}},
$$

где $T_{0}$ - сжимающие усилия. Обратим внимание, что параметр $\lambda$ зависит от сжимающих усилий $T_{0}$.

1. Постановка задачи. Рассмотрим дифференциальное уравнение (5):

$$
(1+\alpha) w^{I V}+4 \beta^{4} w+k_{1} w_{+}+k_{2} w_{-}=-\lambda w^{\prime \prime}
$$


и найдём минимальное значение $\lambda$, соответствующее минимальной нагрузке, при котором краевая задача с граничными условиями шарнирного опирания

$$
w(0)=w(\pi)=0 ; \quad w^{\prime \prime}(0)=w^{\prime \prime}(\pi)=0
$$

имеет нетривиальное решение.

Проинтегрируем по частям функционал, образованный уравнением (7), умноженный на $w(\xi)$. Имеем

$$
\begin{aligned}
&(1+\alpha) \int_{0}^{\pi} w^{\prime \prime 2} d \xi+4 \beta^{4} \int_{0}^{\pi} w^{2} d \xi+k_{1} \int_{0}^{\pi} w_{+}^{2} d \xi+k_{2} \int_{0}^{\pi} w_{-}^{2} d \xi= \\
&=\lambda \int_{0}^{\pi} w^{\prime 2} d \xi .
\end{aligned}
$$

Заменим формулу (8) приближённой с использованием дискретного представления функции $w$, задаваемой её значениями на равномерной сетке, т. е. $w_{i}=w\left(x_{i}\right), i=0,1, \ldots, n$.

Аппроксимируем производные конечно-разностными схемами

$$
w_{i}^{\prime}=\frac{w_{i+1}-w_{i-1}}{2 h} ; \quad w_{i}^{\prime \prime}=\frac{w_{i+1}-2 w_{i}+w_{i-1}}{h^{2}} .
$$

Интегралы будем вычислять по квадратурной формуле трапеций:

$$
\int_{0}^{\pi} f(\xi) d \xi=\frac{\pi}{n}\left(\frac{f\left(\xi_{0}\right)}{2}+f\left(\xi_{1}\right)+f\left(\xi_{2}\right)+\ldots+f\left(\xi_{n-1}\right)+\frac{f\left(\xi_{n}\right)}{2}\right) .
$$

Значения срезок функции в узлах сетки представляем формулами

$$
w_{+}\left(\xi_{i}\right)=b_{i} w_{i}, \quad w_{-}\left(\xi_{i}\right)=\left(1-b_{i}\right) w_{i}, \quad b_{i}= \begin{cases}1, & w_{i}>0 \\ 0, & w_{i} \leqslant 0\end{cases}
$$

Граничные условия шарнирного опирания аппроксимируем формулами

$$
w_{-1}=w_{n+1}=0, \quad w_{0}=w_{1} / 2, \quad w_{n}=w_{n-1} / 2 .
$$

После преобразования уравнение (7) примет вид

$$
A \tilde{w}+C \tilde{w}=\lambda Q \tilde{w}
$$

Таким образом, необходимое условие минимума функционала имеет вид

$$
A \tilde{w}+C \tilde{w}-\lambda Q \tilde{w}=0
$$

где матрицы $A, Q$ - пятидиагональные:

$$
A=\frac{1+\alpha}{h^{3}}\left[\begin{array}{rrrrrrr}
3.25 & -3.5 & 1 & & & & \\
-3.5 & 6 & -4 & 1 & & & \\
1 & -4 & 6 & -4 & 1 & & \\
& . & . & . & . & . & \\
& & 1 & -4 & 6 & -4 & 1 \\
& & & 1 & -4 & 6 & -3.5 \\
& & & & 1 & -3.5 & 3.25
\end{array}\right]
$$




$$
\begin{aligned}
& Q=\frac{1}{4 h}\left[\begin{array}{rrrrrrr}
2.75 & -0.5 & -1 & & & & \\
-0.5 & 2 & 0 & -1 & & & \\
-1 & 0 & 2 & 0 & -1 & & \\
& . & . & . & . & . & \\
& & -1 & 0 & 2 & 0 & -1 \\
& & & -1 & 0 & 2 & -0.5 \\
& & & & -1 & -0.5 & 2.75
\end{array}\right] \text {; } \\
& C=\operatorname{diag}\left[k_{1} b_{i}+k_{2}\left(1-b_{i}\right)+4 \beta^{4}\right] h \text {. }
\end{aligned}
$$

2. Комбинированный алгоритм. Для решения задачи будем использовать комбинированный алгоритм, который включает в себя применение на первой стадии алгоритма полного перебора вариантов (ППВ), а на последующих локального перебора вариантов (ЛПВ) [6].

Для построения части собственного спектра уравнения применяется алгоритм ППВ форм изгиба, который заключается в следующем:

i) перебираются все $2^{m-1}$ возможных представления вектора формы

$$
b=\left[b_{1}, b_{2}, \ldots, b_{m-1}\right]^{\top}
$$

ii) для каждого варианта вектора формы решается задача на собственные значения;

iii) запоминается собственная пара (число и форма), для которой форма изгиба согласуется с выбранным вектором формы.

В процессе применения алгоритма ППВ получаем качественно адекватную форму, то есть собственную форму, имеющую устойчивый с ростом $m$ вид графика.

После этого будем применять алгоритм ЛПВ, используя в качестве приближения полученную качественно адекватную форму:

j) последовательно удваиваем число узлов сетки путём деления интервалов пополам;

jj) осуществляем перебор вариантов лишь вблизи корней последнего приближения к искомой собственной форме;

jjj) процесс продолжается до тех пор, пока соответствующее собственное значение не стабилизируется с требуемой точностью.

Проблема нахождения собственных пар для каждого вектора формы решалась с использованием QR-алгоритма, описанного в [8].

Исходная задача

$$
A \tilde{w}+C \tilde{w}-\lambda Q \tilde{w}=0
$$

приводится к виду

$$
Q^{-1}(A+C) \tilde{w}=\lambda \tilde{w} .
$$

Введём следующее обозначение $\tilde{A}=Q^{-1}(A+C)$. Здесь $\tilde{A}$ - симметричная положительно определённая матрица, значит, она имеет вещественные собственные числа.

В соответствии с теоремами 3.2.20 и 3.2.46 из [7] реализуется следующий итерационный процесс:
1) $\tilde{A}^{0}=\tilde{A}$;
2) $Q^{(k)} R^{(k)}=\tilde{A}^{(k-1)} \tilde{A}^{(k)}=R^{(k)} Q^{(k)}$. 
Все полученные матрицы $Q^{(i)}$ перемножаются. В результате столбцы полученной матрицы будут представлять собой собственные векторы, а на диагонали последней матрицы $\widetilde{A}^{(k)}$ будут находиться собственные числа.

Таким образом с использованием QR-алгоритма на каждом шаге комбинированного алгоритма возможно эффективное отыскание собственных пар.

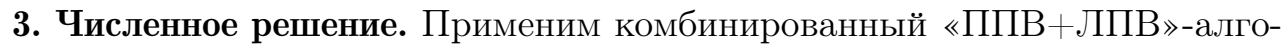
ритм (ППВ при $n=6$, ЛПВ при $n=24)$ при $k_{1}=16$ и $k_{2}=18$. Значения параметра $\alpha$ будем изменять в пределах от 0 до 90. Зафиксируем значение параметра $4 \beta^{4}=43.68$ и согласно формуле (6) получим следующие значения.

\begin{tabular}{c|c|c|c|c|c|c}
\hline$\alpha$ & 0 & 10 & 30 & 50 & 70 & 90 \\
\hline$\lambda$ & 17.5 & 61.7 & 91.9 & 112.1 & 132.3 & 152.5 \\
\hline
\end{tabular}

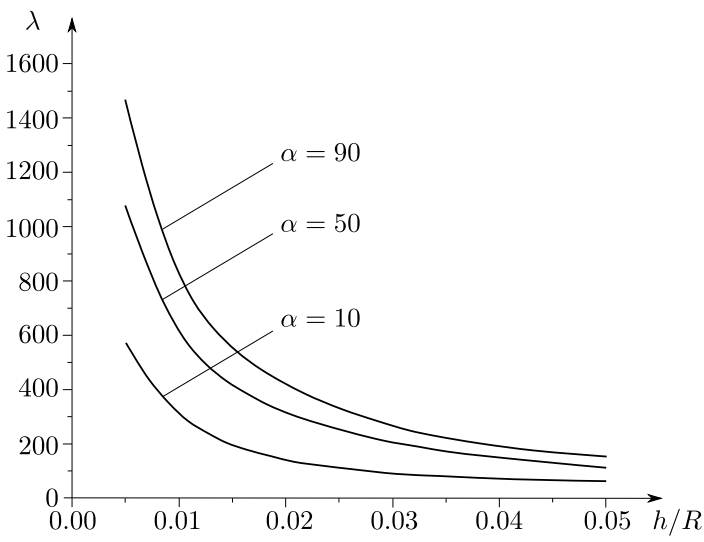

Из полученных значений видно, что при увеличении параметра $\alpha$ ( $\alpha=0$ - оболочка, не подкреплённая стрингерами) прочность оболочки повышается, т. к. увеличивается значение $\lambda$, а значит, и значение первой критической силы.

Фиксируя значения $L$ и $\nu$, получаем зависимость $\lambda$ от $h / R$ и $\alpha$. На рисунке приведены графики зависимости $\lambda$ от $h / R$ при $\alpha=10,50$ и $90(L=200$ cм, $\nu=0.3)$.

Заключение. В результате проведённого численного эксперимента видно, что при увеличении параметра $\alpha$ и при уменьшении $h / R$ значение $\lambda$ увеличивается, в результате чего повышается прочность оболочки. Полученные результаты согласуются с результатами, полученными в статье [6].

\section{СПИСОК ЛИТЕРАТУРЫ/ REFERENCES}

1. Е. И. Михайловский, Математические модели механики упругих тел. Сыктывкар: Сыктывкарск. ун-т, 2007. 516 с. [E. I. Mikhailovsky, Matematicheskiye modeli mekhaniki uprugikh tel [Mathematical models of elastic bodies], Syktyvkar, Syktyvkar Univ. Press, 2007, 516 pp. (In Russian)]

2. В. В. Новожилов, К. Ф. Черных, Е. И. Михайловский, Линейнал теория тонких оболочек. Л.: Политехника, 1991. 656 с. [V. V. Novozhilov, K. F. Chernykh, E. I. Mikhailovsky, Lineynaya teoriya tonkikh obolochek [Linear Theory of Thin Shells], Leningrad, Politekhnika, 1991, 656 pp. (In Russian)]

3. А. С. Вольмир, Устойчивость деформируемых систем. М.: Наука, 1969. 984 с. [A. S. Vol'mir, Ustoychivost' deformiruyemykh sistem [Stability of Deformable Systems], Moscow, Nauka, 1969, 984 pp. (In Russian)]

4. А. П. Филин, Элементы теории оболочек. Л.: Стройиздат, 1989. 384 с. [A. P. Filin, Elementy teorii obolochek [Elements of Shells Theory], Leningrad, Stroyizdat, 1989, 384 pp. (In Russian)]

5. Е. И. Михайловский, Элементы конструктивно-нелинейной механики. Сыктывкар: Сыктывкарск. ун-т, 2011. 201 с. [E. I. Mikhailovsky, Elementy konstruktivno-nelineynoy mekhaniki [Elements of constructive nonlinear mechanics], Syktyvkar, Syktyvkar Univ. Press, 2011, 201 pp. (In Russian)] 
6. Е. И. Михайловский, Е. В. Тулубенская, “Алгоритм локального перебора вариантов в одной существенно нелинейной спектральной задаче" // ПММ, 2010. Т. 74, № 2. С. 299310; Ye. I. Mikhailovskii, Yu. V. Tulubenskaya, "An algorithm for the local exhaustive search for alternatives in an essentially non-linear eigenvalue problem", J. Appl. Math. Mech., 2010, vol. 74, no. 2, pp. 214-222 doi: 10.1016/j.jappmathmech.2010.05.012.

7. D. S. Watkins, Fundamentals of matrix computations, New York, John Wiley \& Sons, 2002, xiii+620 pp. doi: 10.1002/0471249718.

8. M. Panju, "Iterative Methods for Computing Eigenvalues and Eigenvector", The Waterloo Mathematics Review, 2011, vol. 1, pp. 9-19, arXiv: 1105.1185 [math.NA].

Поступила в редакцию 26/XI/2013;

в окончательном варианте - 24/XII/2013;

принята в печать - 19/III/2014.

MSC: 74B20; 74K25, 74K05

\title{
A LONGITUDINAL STABILITY OF A RIBBED COVER IN A MULTIMODULUS ELASTIC MEDIUM
}

\author{
A. Yu. Korablev, E.I. Mikhailovsky, \\ E. V. Tulubenskaya, N. A. Belyaeva \\ Syktyvkar State University, \\ 55, Oktyabr'skiy pr., Syktyvkar, 167001, Russian Federation.
}

The stability of a longitudinal compressed hinge-supported cylindrical cover stiffened by stringers and located on the border of two Winkler's ambiences is considered. The derivation of the equations was carried out under the assumptions: using a simplified theory of Donnell-Vlasov, axisymmetric deformation of a cover, only normal load acts on the shell. The problem is solved using a combined exhaustive search algorithm. This method includes full and local search of variants to search a form deflection and a critical force. Full search of variants is required to construct a form deflection of a shell. Local search of variants is necessary to clarify a critical force. As a result of numerical experiments we found out that increasing the number of stringers reinforces the shell. These results are consistent with the results obtained in the other works.

Keywords: stability, hinge-supported cylindrical cover, combined exhaustive search algorithm.

Received 26/XI/2013;

received in revised form $24 / \mathrm{XII} / 2013$;

accepted 19/III/2014.

ISSN: 2310-7081 (online), 1991-8615 (print); doi: http://dx.doi.org/10.14498/vsgtu1278 (C) 2014 Samara State Technical University.

Citation: A. Yu. Korablev, E. I. Mikhailovsky, E. V. Tulubenskaya, N. A. Belyaev a, "A Longitudinal Stability of a Ribbed Cover in a Multimodulus Elastic Medium", Vestn. Samar. Gos. Tekhn. Univ., Ser. Fiz.-Mat. Nauki [J. Samara State Tech. Univ., Ser. Phys. \& Math. Sci.], 2014, no. 2 (35), pp. 89-95. doi: 10.14498/vsgtu1278. (In Russian)

Author Details: Anatoly Yu. Korablev, Postgraduate Student, Dept. of Mathematical Modeling and Cybernetics. Eugeny I. Mikhailovsky (12.07.1937-11.07.2013) (Dr. Phys. \& Math. Sci.). Elena V. Tulubenskaya (Cand. Phys. \& Math. Sci.), Associate Professor, Dept. of Mathematical Modeling and Cybernetics. Nadezhda A. Belyaeva (Dr. Phys. \& Math. Sci.), Professor, Dept. of Mathematical Modeling and Cybernetics.

E-mail addresses: astroori@mail.ru (A.Yu. Korablev, Corresponding author), vetamile@rambler.ru (E.V. Tulubenskaya), belyayevana@mail.ru (N.A. Belyaeva) 